\title{
Erratum to: Machine Learning on Big Data: A Developmental Approach on Societal Applications
}

Le Hoang Son, Hrudaya Kumar Tripathy, Biswa Ranjan Acharya, Raghvendra Kumar and Jyotir Moy Chatterjee

\section{Erratum to:}

Chapter "Machine Learning on Big Data: A Developmental Approach on Societal Applications" in: M. Mittal et al. (eds.), Big Data Processing Using Spark in Cloud, Studies in Big Data 43, https://doi.org/10.1007/978-981-13-0550-4_7

In the original version of the book, the co-author name "Acharya Biswa Ranjan" in the chapter "Machine Learning on Big Data: A Developmental Approach on Societal Applications" has been changed as "Biswa Ranjan Acharya".

The updated online version of this chapter can be found at https://doi.org/10.1007/978-981-13-0550-4_7 